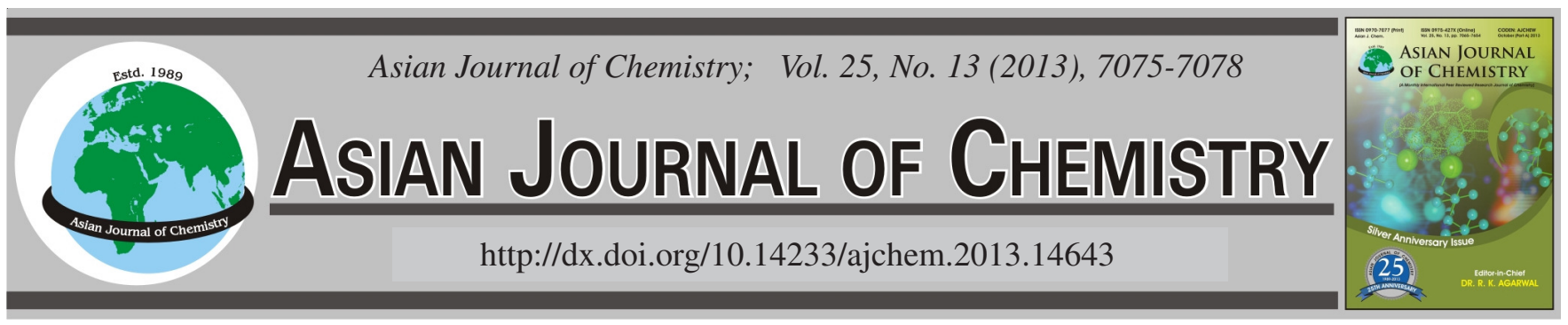

MINI REVIEW

\title{
Flavonoids of the Genus of Acer
}

\author{
WeI LiU ${ }^{1, *}$, YAN OUYANG $^{1}$ and ChUn-PENG WAN ${ }^{2, *}$
}

${ }^{1}$ College of Chemistry and Biological Science, Yili Normal University, Yining 835000, P.R. China

${ }^{2}$ College of Agronomy, Jiangxi Agricultural University, Nanchang 330045, P.R. China

*Corresponding author: E-mail: lemonwan@126.com; nculiuwei@126.com

(Received: 3 October 2012;

Accepted: 12 June 2013)

AJC-13645

\begin{abstract}
The genus of Acer, usually known as maple, is an important genus of the family Aceraceae. Some Acer plants (i.e., red maple, sugar maple and black maple) are used to tapping sap, which were thermally evaporated to produce maple syrup in North America. There are $c a .200$ Acer species widely distributed in Asia, North America and Europe regions. More than 200 chemical constituents have been isolated and identified from nearly 30 Acer species, including flavonoids, tannins, diarylheptanoids, phenyl propanoids, phenylethanoid glycosides, terpenoids, phytosterols, benzoic acid derivatives and other compounds. Among those constituents, flavonoids and tannins were two major and characteristic constituents existing in Acer species. Herein we made a mini-review about the flavonoids constituents from the Acer plants. A checklist of 60 flavonoids from the genus is given and 42 references are cited.
\end{abstract}

Key Words: Acer, Flavonoids, Chemical constituents.

\section{INTRODUCTION}

The genus Acer (Aceraceae), commonly known as maple, contains $c a .200$ species, which were widely distributed in Asia, North America and Europe regions ${ }^{1}$. Some species are used as folk medicine in China and North America. For example, the red maple (Acer rubrum L.) was used to cure the sore eyes by native peoples of eastern Canada and treat various pains and diseases ${ }^{2}$, Acer truncatum Bunge was used as a Chinese folk medicine for the treatment of coronary artery cirrhosis, cerebrovascular diseases and angina pectoris ${ }^{3}$, Acer ginnala was used to treat acute diarrhea and tonsillitis ${ }^{4}$. Extensive studies of the chemical components of Acer have led to the identification more than 200 chemical constituents from nearly 30 Acer species, including flavonoids, tannins, diarylheptanoids, phenyl propanoids, phenylethanoid glyco- sides, terpenoids, phytosterols, benzoic acid derivatives and other compounds. In this review, we will summarize the flavonoids constituents progress referring to 21 species from the genus Acer (Table-1) and list all the 60 flavonoids compounds isolated over the past few decades (Tables 2-5).

Flavonoids constituents: Flavonoids are the major components of this genus. 60 flavonoids compounds were isolated and identified from 21 Acer species over the past few decades, including the flavonol and flavanonol, anthocyanidin, flavone and flavanone, flavan-3-ol, isoflavone and chalone. The main aglycones of these compounds were apigenin, naringenin and luteolin (flavones), kaempferol, quercetin, myrecetin and isorhamnetin (flanonols). The main glycosyls were L-rhamnose, D-glucose, D-galacotose, L-arabinose and D-xylose. Flavonols (glycosides of quercetin, kaempferol and isorhamnetin) were abundant in many Acer species (Figs. 1 and 2).

TABLE-1

FLAVONOIDS INVESTIGATION OF 21 SPECIES FROM THE GENUS Acer

\begin{tabular}{|c|c|c|c|c|c|}
\hline Species & Designation & Species & Designation & Species & Designation \\
\hline A. diabolicum & $\mathrm{A}$ & A. mono & $\mathrm{H}$ & A. rubrum & $\mathrm{O}$ \\
\hline A. barbinerve & B & A. nikoense & I & A. saccharum & $\mathrm{P}$ \\
\hline A. cissifolium & $\mathrm{C}$ & A. negundo & $\mathrm{J}$ & A. aizuense & Q \\
\hline A. ginnala & $\mathrm{D}$ & A. okamotoanum & $\mathrm{K}$ & A. truncatum & $\mathrm{R}$ \\
\hline A. glabrum & $\mathrm{E}$ & A. marmoratum & $\mathrm{L}$ & A. oblongum & $\mathrm{S}$ \\
\hline A. macrophyllum & $\mathrm{F}$ & A. palmatum & M & A. tegmentosum & $\mathrm{T}$ \\
\hline A. mandshuricum & $\mathrm{G}$ & A. platanoides & $\mathrm{N}$ & A. carpinifolium & $\mathrm{U}$ \\
\hline
\end{tabular}




\begin{tabular}{|c|c|c|c|}
\hline \multicolumn{4}{|c|}{$\begin{array}{c}\text { TABLE-2 } \\
\text { FLAVONOLS ISOLATED FROM THE GENUS OF Acer }\end{array}$} \\
\hline No. & Name & Species & Ref. \\
\hline 1 & Quercetin & $\mathrm{C}, \mathrm{D}, \mathrm{H}, \mathrm{I}, \mathrm{R}, \mathrm{S}$ & $3-11$ \\
\hline 2 & Quercetin-3-O- $\beta$-D-arabinoside & $\mathrm{E}, \mathrm{R}$ & $3,12,13$ \\
\hline 3 & Quercetin-3-O- $\beta$-D-galactoside & B, J, K, R, T & 13-18 \\
\hline 4 & Quercetin-3-O- $\alpha$-L-rhamnoside & A-E, H, I, K, L, O, P, R, T, U, & $3,5,7,9,10,12-23$ \\
\hline 5 & Quercetin-3-O- $\beta$-D-glucoside & B-E, G, H, J, O, T & $5,9,10,12,15,17,18,20,24,25$ \\
\hline 6 & Quercetin-3-O-neohesperidoside & $\mathrm{G}$ & 24 \\
\hline 7 & Rutin & $\mathrm{D}, \mathrm{E}, \mathrm{J}, \mathrm{O}$ & $9,12,20,25$ \\
\hline 8 & Quercetin-3-O-[ $\beta$-D-xyloside-( $1 \rightarrow 2)-\beta$-D-glucoside] & $\mathrm{T}$ & 18 \\
\hline 9 & 2"-O-Galloylquercitrin & $\mathrm{D}, \mathrm{O}$ & $9,22,23$ \\
\hline 10 & 3"-O-Galloylquercitrin & $\mathrm{D}, \mathrm{O}$ & 22,23 \\
\hline 11 & Quercetin-3-O-(2"-Galloyl)- $\alpha$-L-arabinoside & $\mathrm{K}$ & 14 \\
\hline 12 & Quercetin-3-O-(2"-Galloyl)- $\beta$-D-galactoside & $\mathrm{K}$ & 14 \\
\hline 13 & Quercetin-3-O-(2", 6"-di-Galloyl)- $\beta$-D-galactoside & $\mathrm{K}$ & 14 \\
\hline 14 & Isorhamnetin & $\mathrm{E}$ & 12 \\
\hline 15 & Isorhamnetin-3-O- $\beta$-D-arabinoside & $\mathrm{R}$ & 3,13 \\
\hline 16 & Isorhamnetin-3-O- $\alpha$-L-rhamnoside & $\mathrm{R}$ & 3 \\
\hline 17 & Isorhamnetin-3-O-rutinoside & $\mathrm{J}$ & 25,26 \\
\hline 18 & Kaempferol & $\mathrm{C}, \mathrm{G}, \mathrm{S}$ & $6,10,24$ \\
\hline 19 & Kaempferol-3-O- $\alpha$-L-rhamnoside & $\mathrm{A}, \mathrm{C}, \mathrm{K}, \mathrm{L}, \mathrm{O}, \mathrm{R}, \mathrm{T}, \mathrm{U}$ & $3,10,13-15,18,20$ \\
\hline 20 & Kaempferol-3-O- $\beta$-D-galactoside & E, J, O & $12,15,20$ \\
\hline 21 & Kaempferol-3-O- $\beta$-D-glucoside & $\mathrm{G}, \mathrm{J}, \mathrm{O}, \mathrm{R}$ & $16,20,24,25$ \\
\hline 22 & Kaempferol-3-O- $\alpha$-L-arabinoside & $\mathrm{K}$ & 14 \\
\hline 23 & Kaempferol-3-O-neohesperidoside & G & 24 \\
\hline 24 & Kaempferol-3-O-rutinoside & $\mathrm{J}, \mathrm{O}$ & 20,26 \\
\hline 25 & Myricetin-3-O- $\alpha$-L-rhamnoside & $\mathrm{R}, \mathrm{T}$ & $3,13,18$ \\
\hline 26 & 6-Hydroxy-quercetin-3-O-galactoside & $\mathrm{T}$ & 18 \\
\hline 27 & 5,3'-Dihydroxy-3,7,4'-trimethoxy flavone & $\mathrm{T}$ & 27 \\
\hline 28 & 3,7,3',4'-Tetramethyl-quercetin & $\mathrm{T}$ & 27 \\
\hline 29 & Kaemferol-3,7-di-glucoside & $\mathrm{J}$ & 26 \\
\hline
\end{tabular}

\begin{tabular}{|c|c|c|c|}
\hline \multicolumn{4}{|c|}{$\begin{array}{c}\text { TABLE-3 } \\
\text { ANTHOCYANIDINS ISOLATED FROM THE GENUS OF Acer }\end{array}$} \\
\hline No. & Name & Species & Ref. \\
\hline 30 & Cyanidin-3-O- $\beta$-glucoside & $\mathrm{M}, \mathrm{N}$ & $28-30$ \\
\hline 31 & Cyanidin-3-O-(2"-Galloyl- $\beta$-glucoside) & $\mathrm{N}$ & 28,31 \\
\hline 32 & Cyanidin-3-O-(2", 3"-di-Galloyl- $\beta$-glucoside) & $\mathrm{N}$ & 28 \\
\hline 33 & Cyanidin-3-O-rutinoside & M & 29,30 \\
\hline 34 & Cyanidin-3-O-[2"-O-(Galloyl)-6"-O-( $\alpha$-L-rhamnoside)- $\beta$-D-glucoside] & $\mathrm{N}$ & 31 \\
\hline 35 & Cyanidin-3-O-[2"-O-( $\beta$-D-xyloside)- 6 "-O-(a-L-rhamnoside)- $\beta$-D-glucoside] & $\mathrm{F}$ & 32 \\
\hline 36 & Delphinidin & Q & 33 \\
\hline 37 & Delphinidin-3,5-di-glucoside & $\mathrm{Q}$ & 33 \\
\hline
\end{tabular}

\begin{tabular}{|c|c|c|c|}
\hline \multicolumn{4}{|c|}{$\begin{array}{c}\text { TABLE-4 } \\
\text { FLAVONES AND FLAVANONES ISOLATED } \\
\text { FROM THE GENUS OF Acer }\end{array}$} \\
\hline No. & Name & Species & Ref. \\
\hline 38 & Apigenin & M, S & 6,34 \\
\hline 39 & Apigenin-8-C- $\beta$-D-glucoside & M & 34,35 \\
\hline 40 & Apigenin-6-C- $\beta$-D-glucoside & M & 34 \\
\hline 41 & Luteolin-8-C- $\beta$-D-glucoside & M & 34 \\
\hline 42 & Luteolin-6-C- $\beta$-D-glucoside & M & 34 \\
\hline 43 & Luteolin-4'-O- $\beta$-D-glucoside & $\mathrm{C}$ & 36 \\
\hline 44 & Naringenin & $\mathrm{H}$ & 5 \\
\hline 45 & Naringenin-7-O- $\beta$-D-glucoside & $\mathrm{H}$ & 5 \\
\hline 46 & Eriodictyol & $\mathrm{H}$ & 5 \\
\hline 47 & Eriodictyol-7-O- $\beta$-D-glucoside & $\mathrm{H}$ & 5 \\
\hline
\end{tabular}

Flavonol and flavanonol: Twenty nine flavonols (1-29) have been reported from most species of this genus except four species (i.e., A. macrophyllum, A. palmatum, A. platanoides and A. aizuense). Quercetin and kaempferol were two major aglycones, the rhamnoside of quercetin (4) and kaempferol (19) were appearance in many Acer species. It is supposed that the two components were characteristic for this genus. It is noteworthy that some species (i.e., A. ginnala, $A$. rubrum and A. okamotoanum) of the genus contain large amounts of Galloyl substituent of quercetin glycosides (9-13) $)^{9,14,22,23}$. Two polymethoxyflavones (PMF, 27 and 28) were isolated from A. tegmentosum ${ }^{27}$ and kaemferol-3,7-diglucoside (29) were isolated from A. negundo ${ }^{26}$. Two flavanonols dihydromyricetin (48) and morin-3-O- $\alpha-\mathrm{L}-$ xyloside (49) were isolated from A. tegmentosum ${ }^{18,27}$.

Anthocyanidin: Anthocyanidins are the pigment compositions in Acer species. Eight anthocyanidin (30-37) compounds were isolated from four Acer species (i.e., A. macrophyllum, A. palmatum, A. platanoides and A. aizuense). Cyanidin and delphinidin were the aglycones of those anthocyanidins $\mathrm{s}^{28-33}$. 
TABLE-5

FLAVAN-3-OL ISOLATED FROM THE GENUS OF Acer

\begin{tabular}{clcc}
\hline No. & \multicolumn{1}{c}{ Name } & Species & Ref. \\
\hline 50 & Catechin & B, G, I, O, T & T, 17, 18, 20, 23, 24, 37-42 \\
51 & Gallocatechin & $\mathrm{T}$ & 18 \\
52 & (+)-Catechin-3-O-(3,4- & $\mathrm{T}$ & 18 \\
53 & dihydroxybenzoyl) & B, I, O & $17,20,23,40,41$ \\
54 & Epicatechin & B, O & 17,23 \\
55 & Epicatechin-3-O-gallate & O & 42 \\
56 & Procyanidin dimer & O & 42 \\
57 & Procyanidin trimer & O & 23 \\
58 & Procyanidin A2 & O & 23 \\
\hline
\end{tabular}<smiles>[R]c1cc(-c2oc3cc(O)cc(O)c3c(=O)c2O)cc(Br)c1O</smiles><smiles></smiles>

\begin{tabular}{|c|c|c|c|c|c|c|c|}
\hline No. & $\mathrm{R}_{1}$ & $\mathrm{R}_{2}$ & $\mathrm{R}_{3}$ & No & $\mathrm{R}_{1}$ & $\mathrm{R}_{2}$ & $\mathrm{R}_{3}$ \\
\hline 1 & $\mathrm{OH}$ & $\mathrm{H}$ & $\mathrm{H}$ & 30 & $\mathrm{H}$ & $\beta$-D-Glu & $\mathrm{H}$ \\
\hline 2 & $\mathrm{OH}$ & $\mathrm{H}$ & $\beta$-D-Ara & 31 & $\mathrm{H}$ & $\beta$-D-Glu(2"-O-Galloyl) & $\mathrm{H}$ \\
\hline 3 & $\mathrm{OH}$ & $\mathrm{H}$ & $\beta$-D-Gal & 32 & $\mathrm{H}$ & $\beta$-D-Glu(2", 6"-di-O-Galloyl) & $\mathrm{H}$ \\
\hline 4 & $\mathrm{OH}$ & $\mathrm{H}$ & $\alpha$-L-Rha & 33 & $\mathrm{H}$ & Rutinoside & $\mathrm{H}$ \\
\hline 5 & $\mathrm{OH}$ & $\mathrm{H}$ & $\beta$-D-Glu & 34 & $\mathrm{H}$ & $\alpha$-L-Rha(2"-O-Galloyl) $(1 \rightarrow 6)-\beta$-D-Glu & $\mathrm{H}$ \\
\hline 6 & $\mathrm{OH}$ & $\mathrm{H}$ & neohesperidoside & 35 & $\mathrm{H}$ & $\beta$-D-Xyl $(1 \rightarrow 2)-\alpha-L-R h a(1 \rightarrow 6)-\beta$-D-Glu & $\mathrm{H}$ \\
\hline 7 & $\mathrm{OH}$ & $\mathrm{H}$ & Rutinoside & 36 & $\mathrm{OH}$ & $\mathrm{H}$ & $\mathrm{H}$ \\
\hline 8 & $\mathrm{OH}$ & $\mathrm{H}$ & $\beta$-D-Xyl- $(1 \rightarrow 2)-\beta-D-G l u$ & 37 & $\mathrm{OH}$ & $\beta$-D-Glu & $\beta$-D-Glu \\
\hline
\end{tabular}

$\begin{array}{llll}8 & \mathrm{OH} & \mathrm{H} & \beta-\mathrm{D}-\mathrm{Xyl}-(1 \rightarrow 2)-\beta-\mathrm{D}-\mathrm{Glu} \\ 9 & \mathrm{OH} & \mathrm{H} & \alpha-\mathrm{L}-\mathrm{Bh}(2 "-\mathrm{O}-\mathrm{G} a \mathrm{lloyl})\end{array}$

$\begin{array}{lll}\mathrm{OH} & \mathrm{H} & \alpha \text {-L-Rha(3"-O-Galloyl) }\end{array}$

1 OH $\quad \mathrm{H} \quad \alpha$-L-Ara(2"-O-Galloyl)

$\mathrm{OH} \quad \mathrm{H} \quad \beta$-D-Gal(2"-O-Galloyl)

OH $\quad$ H $\quad \beta$-D-Gal(2",6"-di-O-Galloyl)

$\mathrm{OCH}_{3} \quad \mathrm{H} \quad \mathrm{H}$

$\mathrm{OCH}_{3} \quad \mathrm{H} \quad \beta$-D-Ara

$\mathrm{OCH}_{3} \quad \mathrm{H} \quad \alpha$-L-Rha

$\mathrm{OCH}_{3} \quad \mathrm{H} \quad$ Rutinoside

$\mathrm{H} \quad \mathrm{H} \quad \mathrm{H}$

$\mathrm{H}$

$\alpha$-L-Rha

$\beta$-D-Gal

$\begin{array}{llll}21 & \mathrm{H} & \mathrm{H} & \beta \text {-D-Glu } \\ 22 & \mathrm{H} & \mathrm{H} & \alpha \text {-L-Ara }\end{array}$

\begin{tabular}{l|lll}
21 & H & H & $\beta$-D-Glu \\
22 & H & H & $\alpha$-L-Ara \\
23 & H & H & neohesperidoside
\end{tabular}

$\begin{array}{llll}22 & \mathrm{H} & \mathrm{H} & \alpha \text {-L-Ara } \\ 23 & \mathrm{H} & \mathrm{H} & \text { neohesperidoside }\end{array}$<smiles>[R6]c1cc(C2CC(=O)c3c(O)cc(O)cc3O2)ccc1O</smiles>

$19 \mathrm{H}$

$24 \mathrm{H} \quad \mathrm{H} \quad$ Rutinoside

25 OH OH $\quad \alpha$-L-Rhamnoside<smiles>COc1cc(O)c2c(=O)c(OC)c(-c3ccc(O)cc3)oc2c1</smiles><smiles>O=C1c2c(O)cc(O)cc2OC(c2cc(O)c(O)c(O)c2)C1O</smiles>

48

\begin{tabular}{|c|c|c|c|c|c|c|c|}
\hline NO & $\mathrm{R}_{1}$ & $\mathrm{R}_{2}$ & $\mathrm{R}_{3}$ & $\mathrm{R}_{4}$ & NO & $\mathrm{R}_{1}$ & $\mathrm{R}_{2}$ \\
\hline 38 & $\mathrm{H}$ & $\mathrm{H}$ & $\mathrm{H}$ & $\mathrm{H}$ & 44 & $\mathrm{H}$ & $\mathrm{H}$ \\
\hline 39 & $\mathrm{H}$ & $\mathrm{H}$ & $\beta$-D-Glu & $\mathrm{H}$ & 45 & $\beta$-D-Glu & $\mathrm{H}$ \\
\hline 40 & $\mathrm{H}$ & $\mathrm{H}$ & $\mathrm{H}$ & $\beta$-D-Glu & 46 & $\mathrm{H}$ & $\mathrm{OH}$ \\
\hline 41 & $\mathrm{OH}$ & $\mathrm{H}$ & $\beta$-D-Glu & $\mathrm{H}$ & 47 & $\beta$-D-Glu & $\mathrm{OH}$ \\
\hline
\end{tabular}

$28 \mathrm{R}=\mathrm{CH}_{3}$<smiles>O=C1c2c(O)cc(O)cc2OC(c2ccc(O)cc2O)C1OCCO</smiles>

49

59

Fig. 1. Chemical structure of flavonoids isolated from the genus of Acer 


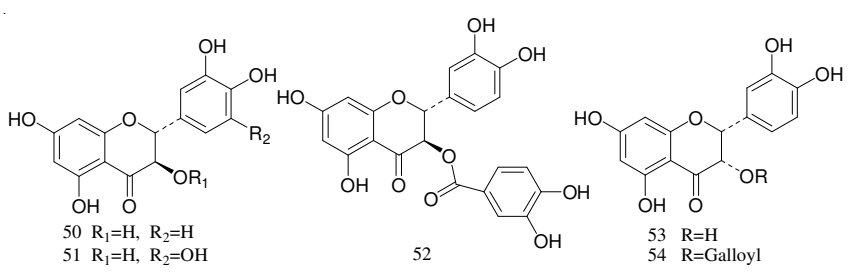<smiles>Oc1cc(O)c2c(c1)OC(c1ccc(O)c(O)c1)C(O)C2c1c(O)cc(O)c2c1OC(c1ccc(O)c(O)c1)C(O)C2O</smiles>

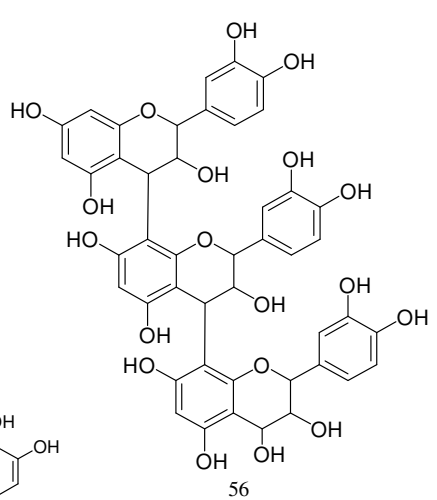

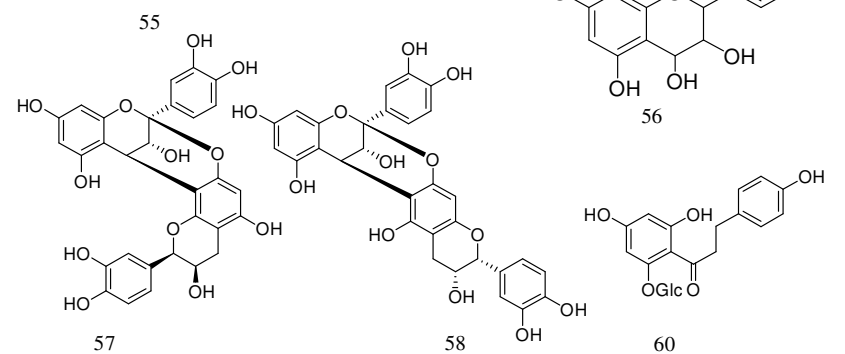

Fig. 2. Chemical structure of flavonoids isolated from the genus of Acer

Flavone and flavanone: Six flavones (38-43) with the aglycones of apigenin and luteolin were isolated from $A$. palmatum, A. oblongum and $A$. cissifolium $^{34-36}$. Compounds 39-42 were four flavone $\mathrm{C}$-glycosides isolated from $A$. palmatum $^{34}$. Four flavanonols (44-47) with the aglycones of naringenin and eriodictyol were isolated from $A$. mono $o^{5}$.

Flavan-3-ol: Nine flavan-3-ol (50-58) were isolated from A. barbinerve, A. mandshuricum, A. rubrum, A. tegmentosum and $A$. nikoense $e^{17,18,20-23,40-42}$. Catechin (50) was appearance in many Acer species and most of the flavan-3-ol compounds were isolated from Acer rubrum. Recently we also had isolated two A-type procyanidins (procyanidin A2 and A6, 57-58) from the stem of Acer rubrum ${ }^{23}$.

Isoflavone and chalone: An isoflavone (isoluteolin, 59) and a chalone (phloridzin, 60) were isolated from $A$. negundo ${ }^{26}$ and the stem of Acer rubrum ${ }^{23}$, respectively.

\section{Conclusion}

The genus Acer (Aceraceae) contains ca. 200 species, which were widely distributed in Asia, North America and Europe regions. Many species of this genus have been used in traditional folk medicine. Extensive studies of the chemical components of Acer have led to the identification more than 200 chemical constituents from nearly 30 Acer species. However, there are still many other Acer species that have not been received or only little attention. Phytochemical and biological studies should focus on these plants from now on in order to fully exploit Acer species.

\section{ACKNOWLEDGEMENTS}

This project was supported by the College Project of Yili Normal University (No. YJZD02).

\section{REFERENCES}

1. D.M. Gelderen, P.C. Jong and H.J. Oterdoom, Maples of the World, Timber Press, Portland, Vol. 1, p. 15 (1994).

2. T. Arnason, R.J. Hebda and T. Johns, Can. J. Bot, 59, 2189 (1981).

3. W.H. Zhao, L.F. Gao, W. Gao, Y.S. Yuan, C.C. Gao, L.G. Cao, Z.Z. Hu, J.Q. Guo and Y.X. Zhang, Nat. Prod. Res., 25, 422 (2011).

4. C. Song, N. Zhang, R. Xu and H. Song, Acta Chim. Sin., 40, 1142 (1982).

5. H. Yang, S.H. Sung and Y.C. Kim, J. Nat. Prod., 68, 101 (2005).

6. N. Parveen, N.U. Khan, T. Inoue and M. Sakurai, Phytochemistry, 27, 3990 (1988).

7. T. Inoue, Y. Ishidate, M. Fujita, M. Kubo, M. Fukushima and M. Nagai, Yakugaku Zasshi, 98, 41 (1978).

8. Y. Li and F. Zeng, Nat. Prod. Res. Develop., 18, 426 (2006).

9. W.Y. Park, Saengyak Hakhoechi, 27, 212 (1996).

10. K. Miyazaki, S. Ishizawa, S. Nagumo, T. Inoue and M. Nagai, Shoyakugaku Zasshi, 45, 333 (1991).

11. C. Song, R. Xu, G. Song, S. Hong, N. Zhang, F. Zhang and Y. Shen, Yaoxue Tongbao, 16, 756 (1981).

12. D.E. Justice, A.R. Reid and B.A. Bohm, Biochem. System Ecol., 23, 263 (1995).

13. B. Xie, F. Xu and L. Li, Acta Botanica Yunnanica, 27, 232 (2005).

14. H.J. Kim, E.R. Woo, C.G. Shin and H. Park, J. Nat. Prod., 61, 145 (1998).

15. M. Aritomi, Yakugaku Zasshi, 84, 360 (1964).

16. X. Huang, L. Tan, K. Gu and C. Li, China J. Chin. Mater. Med., 32, 1544 (2007).

17. Dong-Joo Kwon and Y.-S. Bae, Chem. Nat. Comp., 47, 636 (2011).

18. N.H. Tung, Y. Ding, S.K. Kim, K.H. Bae and Y.H. Kim, J. Agric. Food. Chem., 56, 10510 (2008).

19. W.X. Tian, X.F. Ma, L.H. Wu, X.L. Cao and Y. Ito, J. Chromatogr. A, 1070, 211 (2005)

20. M.M. Abou-Zaid, B.V. Helson, C. Nozzolillo and J.T. Arnason, J. Chem. Ecol., 27, 2517 (2001).

21. T. Hatano, S. Hattori, Y. Ikeda, T. Shingu and T. Okuda, Chem. Pharm. Bull., 38, 1902 (1990).

22. F.L. Hu, R.L. Lu and T. Xia, J. Food Sci., 75, H239 (2010).

23. C. Wan, T. Yuan, M. Xie and N.P. Seeram, Biochem. System Ecol., 44, 1 (2012).

24. Y. Ding, C. Liang, H.T. Nguyen, E.M. Choi, J.A. Kim and Y.H. Kim, Bull. Korean Chem. Soc., 31, 929 (2010).

25. E. Backheet, Bull. Pharm. Sci., 26, 77 (2003).

26. E.Y. Backheet, Bull. Pharm. Sci., 24, 1 (2001)

27. K.M. Park, M.C. Yang, K.H. Lee, K.R. Kim, S.U. Choi and K.R. Lee, Arch. Pharm. Res., 29, 1086 (2006).

28. T. Fossen and M. Andersen, Phytochemistry, 52, 1697 (1999).

29. V. Schmitzer, G. Osterc, R. Veberic and F. Stampar, Sci. Hort., 119, 442 (2009)

30. V. Schmitzer, F. Stampar, R. Veberic and G. Osterc, Acta Physiol. Plant, 31, 415 (2009).

31. S. Ji, N. Saito, M. Yokoi, A. Shigihara and T. Honda, Phytochemistry, 31, 655 (1992).

32. S.B. Ji, M. Yokoi, N. Saito, Y. Uedas, A. Shigihara and T. Honda, Technical Bull. Faculty Horticul.-Chiba Univ. (Japan), 49, 13 (1995).

33. M. Tsujimura and C. Nakahama, Nippon Nogei Kagaku Kaishi, 39, 209 (1965)

34. M. Aritomi, Yakugaku Zasshi, 83, 737 (1963).

35. J.H. Kim, B.C. Lee, G.S. Sim, D.H. Lee, K.E. Lee, Y.P. Yun and H.B. Pyo, Arch. Pharm. Res., 28, 195 (2005).

36. M. Aritomi, Chem. Pharm. Bull., 12, 841 (1964).

37. T. Yonezawa, J.W. Lee, H. Akazawa, M. Inagaki, B.Y. Cha, K. Nagai, K. Yagasaki, T. Akihisa and J.T. Woo, Bioorg. Med. Chem. Lett., 21, 3248 (2011).

38. T. Akihisa, Y. Taguchi, K. Yasukawa, H. Tokuda, H. Akazawa, T. Suzuki and Y. Kimura, Chem. Pharm. Bull., 54, 735 (2006).

39. H. Akazawa, T. Akihisa, Y. Taguchi, N. Banno, R. Yoneima and K. Yasukawa, Biol. Pharm. Bull., 29, 1970 (2006).

40. T. Morikawa, J. Tao, K. Ueda, H. Matsuda and M. Yoshikawa, Chem. Pharm. Bull., 51, 62 (2003).

41. T. Morikawa, J. Tao, I. Toguchida, H. Matsuda and M. Yoshikawa, J. Nat. Prod., 66, 86 (2003).

42. V. Narayana and T.R. Seshadri, Indian J. Chem., 7, 213 (1969). 